\title{
2-MV electrostatic quadrupole injector for heavy-ion fusion
}

\author{
F. M. Bieniosek, C. M. Celata, E. Henestroza, J. W. Kwan, L. Prost, and P. A. Seidl \\ Lawrence Berkeley National Laboratory and Heavy-Ion Fusion Virtual National Laboratory, \\ 1 Cyclotron Road, Berkeley, California 94720, USA \\ A. Friedman, D. P. Grote, and S. M. Lund \\ Lawrence Livermore National Laboratory and Heavy-Ion Fusion Virtual National Laboratory, \\ P.O. Box 808, Livermore, California 94550, USA \\ I. Haber \\ Institute for Research in Electronics and Applied Physics, University of Maryland, College Park, Maryland 20742, USA
}

(Received 11 November 2004; published 19 January 2005)

\begin{abstract}
High current and low emittance are principal requirements for heavy-ion injection into a linac driver for inertial fusion energy. An electrostatic quadrupole injector is capable of providing these high charge density and low emittance beams. We have modified the existing 2-MV injector to reduce beam emittance and to double the pulse length. We characterize the beam delivered by the modified injector to the High Current Transport Experiment and the effects of finite rise time of the extraction voltage pulse in the diode on the beam head. We demonstrate techniques for mitigating aberrations and reducing beam emittance growth in the injector.
\end{abstract}

DOI: 10.1103/PhysRevSTAB.8.010101

\section{INTRODUCTION}

Heavy-ion fusion accelerator (“driver") design architecture generally envisions multiple parallel beam channels with each channel transporting a beam of line charge density of about $0.25 \mu \mathrm{C} / \mathrm{m}$. There are stringent requirements on heavy-ion beam current $(I \geq 0.5 \mathrm{~A}$ for pulse length $\geq 10 \mu \mathrm{s})$ and transverse emittance $\left(\varepsilon_{n}<\right.$ $1 \pi \mathrm{mm} \mathrm{mrad}$ ) at the injector for each beam line ultimately to produce the desired beam spot size and intensity on the fusion target [1].

An electrostatic quadrupole (ESQ) accelerator [2,3], to be distinguished from ESQ transport systems, consists of an axisymmetric front end (a vacuum diode), followed by a column of high voltage quadrupoles which simultaneously focuses and accelerates the beam. At low beam energy and current an electrostatic aperture column (ESAC), which consists of a column of aperture lenses, is commonly used in injector columns. Simple scaling relations [4] show that the transportable current density in an ESAC decreases with beam energy as $V_{\text {beam }}^{-1 / 2}$, but in an ESQ accelerator column, the transportable current density increases with beam energy as $V_{\text {beam }}^{1 / 2}$, where $V_{\text {beam }}$ is the beam ion kinetic energy in electron volts. For beam energy above about $V_{\text {beam }} \sim 1 \mathrm{MeV}$, an ESQ accelerator can transport more current than an equivalent ESAC. The ESQs provide strong focusing which is not directly tied to the longitudinal accelerating field. Secondary electrons are swept out by the transverse focusing fields, leading to relatively low energy electrons and reducing the breakdown risks. The capability for handling high current density allows sources with relatively small transverse size and thus low intrinsic emittance.
PACS numbers: 29.17.+w, 29.27.Ac, 41.85.-p, 07.77.Ka

The 2-MV injector is an ESQ-based accelerator that was developed in 1993 for the Induction Linac Systems Experiment (ILSE)/Elise project [5], and represents the injector for a single heavy-ion fusion driver beam. It delivers a $\mathrm{K}^{+}$beam at energy up to $2 \mathrm{MeV}$ and a beam current up to $\sim 0.8 \mathrm{~A}$. It has been modified to produce a longer beam pulse length with reduced emittance and currently serves as the injector [6,7] for the High Current Transport Experiment (HCX) [8]. HCX explores intense heavy-ionbeam transport in electrostatic- and magnetic-focused quadrupole channels representative of the low energy end of an induction linac driver.

A critical issue for ESQ-based accelerators for this and other applications is the distortion of beam phase space by aberrations introduced by the accelerating structures. In the vacuum diode section, aberrations are due to nonlinearities in the electrostatic field configuration, such as imperfections in the diode geometry, the presence of a switched gate electrode, nonuniform current density at the surface of the source, and edge effects at the interface between the source and the Pierce structure. In the ESQ section nonlinear forces in the focusing system drive emittance growth. One nonlinear effect is the energy effect due to the slowing of the ions by a positively biased ESQ electrode and acceleration by a negatively biased electrode. When the ratio of transverse quadrupole potential $V_{q}$ is a significant fraction of $V_{\text {beam }}$, the resulting S-shaped kinematic distortions across the beam radius lead to emittance growth. This effect has been mitigated in the injector by reducing the beam envelope through the ESQ section, and by increasing the energy of the beam entering the ESQ section as much as practical without risking breakdown in the diode section. 
Another critical issue is the transient behavior of the ion beam pulse. In a one-dimensional geometry, if the rise time of the applied diode voltage pulse is the same as the transit time of the beam ions through the diode, the rise time of the beam current pulse exiting the diode is minimized [9]. Low current at the head of the beam pulse is overfocused by the quadrupoles, leading to transient beam envelope excursions. An enlarged mushroom-shaped beam head that scrapes the wall of the channel may develop. This transient creates a gas and electron cloud that can perturb the main body of the beam pulse. In HCX such a beam head exists and persists through the matching section and transport section of the experiment.

A third critical issue is the source, which must be capable of emitting the required beam current density, with adequate emittance, uniformity, species purity, and lifetime. As noted below, we have utilized the surface ionization source which has been shown capable of meeting these requirements.

This paper presents recent upgrades to the equipment and characterization of the beam in two different experimental configurations during commissioning the injector for the HCX. The critical issues are discussed in a com- parison of experimental results for beam current, density and transverse phase-space distributions with the predictions of particle-in-cell (PIC) simulations.

\section{INJECTOR CONFIGURATION AND DIAGNOSTICS}

\section{A. Injector assembly}

The injector consists of a hot-surface ionization source assembly followed by an extraction diode and by a fourquadrupole ESQ accelerating section. Figure 1 shows the accelerator which consists of the diode section and the ESQ section mounted in the same column. The electron trap is associated with a Rogowski coil diagnostic and is not necessary when the injector is utilized for HCX since the ESQ electrodes in the matching section sweep out electrons from the beam. The diode source assembly, gate (extraction), and Pierce electrodes are shown in the red inset.

The injector is contained inside a pressure vessel that houses the Marx generator, the high voltage dome containing the ion source, extraction pulse equipment and diagnostics, and the ESQ section. The high voltage dome is

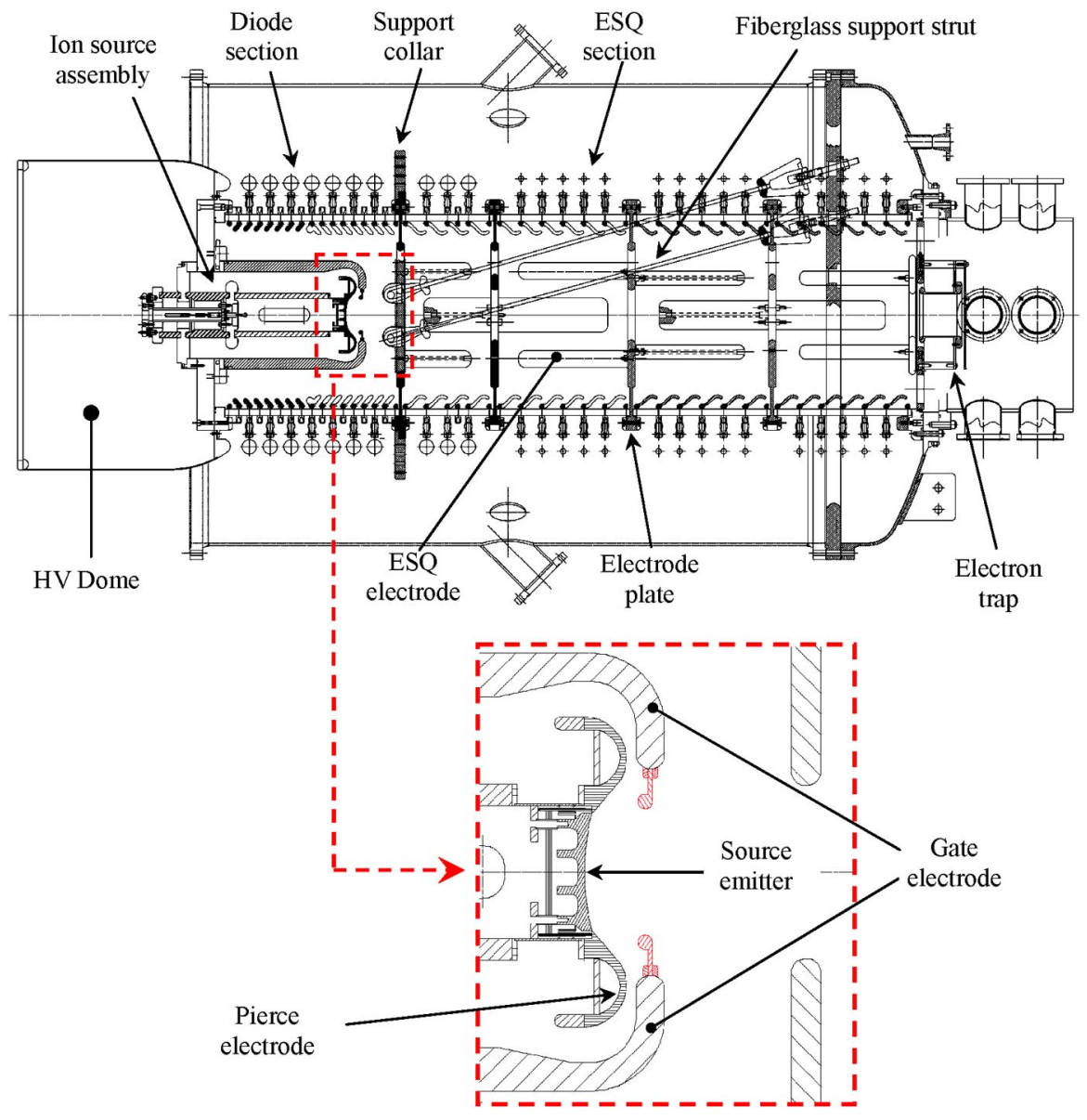

FIG. 1. (Color) 2-MV injector accelerator column showing fiberglass support struts. The column length is $2.4 \mathrm{~m}$. The red part in the insert shows the addition to the gate electrode reducing its aperture diameter from 179.5 to $110 \mathrm{~mm}$. 
electrically powered by a hydraulically driven generator. Controls and diagnostic information are transmitted by fiber-optic link. The accelerator column consists of a series of niobium voltage-grading rings brazed to ceramic insulators. There are four voltage tap points on a resistive voltage divider $(R=5 \mathrm{k} \Omega)$ which consists of a sodium sulfate solution in water in flexible plastic tubes surrounding the column. The voltage taps establish the bias potential for each of the four quadrupoles. The bore radius for the first two quadrupoles (Q1 and Q2) is $r_{b}=12 \mathrm{~cm}$, and for the third and fourth quadrupoles (Q3 and Q4) $r_{b}=$ $10 \mathrm{~cm}$. The ESQ electrodes have a cylindrical inner face with radius of curvature $r_{c}=8 / 7 r_{b}$. The outer faces of the electrodes are truncated to fit inside the accelerator column. The highest surface electric field strength across a vacuum gap is $80 \mathrm{kV} / \mathrm{cm}$, between the Pierce and gate electrodes; the longitudinal accelerating electric field across the ceramic rings of the insulating column ranges from 5 to $11 \mathrm{kV} / \mathrm{cm}$. The pressure vessel provides electrical insulation of the high voltage structure and is filled with a high-pressure (up to $5.3 \mathrm{~atm}$ ) insulating gas mixture $(90 \%$ $\mathrm{N}_{2}, 10 \% \mathrm{SF}_{6}$ ) [10]. The stability of the resistive divider, which is maintained at the $1 \%$ level, depends on the integrity of the plastic tubes and the conductivity of the water resistor. Care must be taken to limit the pressure in the vessel to less than the critical pressure that may collapse the plastic tubes. Further construction details are presented elsewhere [5].

\section{B. Pulsed power}

The modified Marx generator consists of 38 stages, and is capable of delivering $0.8-2 \mathrm{MV}$ to the high voltage dome at charging voltages of $20-50 \mathrm{kV}$. Each stage is a two-section pulse-forming network, initially configured as two independent capacitive-discharge sections, one of which is resistive and the other inductive. This simple network produced a flattop adequate for the ILSE application when matched into the capacitance of the dome and the resistance of the column voltage divider. The injector delivered up to a $2 \mu$ s square beam pulse, limited by the flattop of the Marx voltage pulse.

The HCX project involves, in part, study of beam phenomena which occur on relatively long time scales, e.g., secondary electrons and gas which build up during the beam pulse due to interactions with the wall and ambient gas [11]. Therefore it is advantageous to lengthen the beam pulse as much as is practical. It proved to be possible to double the beam pulse length without modification to the existing Marx circuit components by introducing an aircore magnetic coupling transformer to couple the large inductance $\mathrm{L}_{1}$ of the inductive section of the Marx generator stages to a small inductance $\mathrm{L}_{2}$ in the resistive section (Fig. 2). Increasing the coupling coefficient $k_{12}$ between $\mathrm{L}_{1}$ and $\mathrm{L}_{2}$ flattens the pulse up to an optimal coupling coefficient of $k_{12} \approx 0.32$ for $\mathrm{L}_{1}=440 \mu \mathrm{H}$ and $\mathrm{L}_{2}=5 \mu \mathrm{H}$.

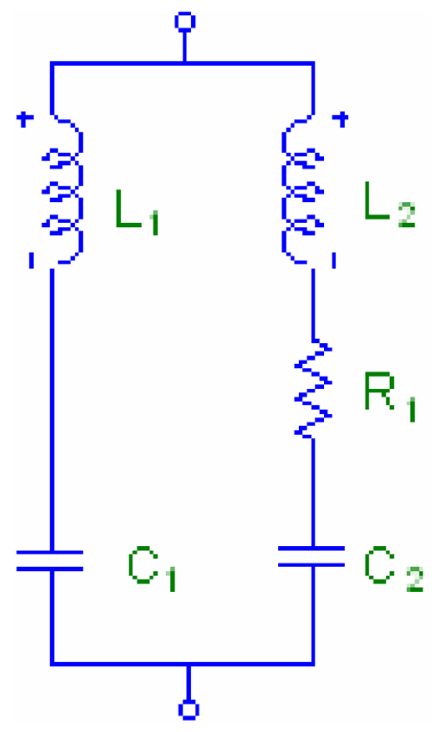

FIG. 2. (Color) Circuit drawing of each of 38 modified Marx generator stages. Here $\mathrm{C}_{1}=\mathrm{C}_{2}=40 \mathrm{nF}, \mathrm{R}_{1}=50 \Omega, \mathrm{L}_{1}=$ $440 \mu \mathrm{H}, \mathrm{L}_{2}=5 \mu \mathrm{H}$, and the coupling between $\mathrm{L}_{1}$ and $\mathrm{L}_{2}$ is $\mathrm{M}_{12}=k_{12}\left(\mathrm{~L}_{1} \mathrm{~L}_{2}\right)^{1 / 2}=15 \mu \mathrm{H}$.

Further increases in magnetic coupling continue to lengthen the pulse, but it begins to exhibit a double peak. At the optimal amount of coupling, the length of the flattop (flat to within $<1 \%$ of peak value) increases from 3.2 to $5.2 \mu \mathrm{s}$. Allowing for beam transit times in the diode and ESQ accelerator, the modification allowed the extracted beam pulse flattop length to be doubled, from 2 to $4 \mu \mathrm{s}$, once the length of the extraction pulser pulse forming network (PFN) was extended.

The source is dc reverse biased at a negative potential $-V_{\text {bias }}$, as much as $-60 \mathrm{kV}$, with respect to the gate electrode in order to inhibit ion emission. Ion extraction is provided by an extraction pulser that rapidly switches the source-gate voltage into forward bias. The rise time of the beam current is minimized in this way, since the extraction pulser load is limited to the capacitance of the source assembly, rather than the full diode. The extraction pulser is a tunable 6-stage PFN coupled to the source assembly through a step-up output transformer. During ion extraction, the extraction pulser delivers up to $V_{\text {gate }}=140 \mathrm{kV}$ with rise time (10\%-90\%) of $0.4 \mu \mathrm{s}$ and a flattop ( $\pm 1 \%$ ) of $4.3 \mu \mathrm{s}$. During extraction (Fig. 3), the source voltage swings from its initial value of $-V_{\text {bias }}$ to the extraction voltage $V_{\text {ext }}=V_{\text {gate }}-V_{\text {bias }}$, up to $80 \mathrm{kV}$. Note that the extraction pulse must be longer than the desired beam flattop by the beam transit time in the diode, about $0.35 \mu$ s.

\section{Voltage calibration}

The accelerating voltage is the sum of the Marx generator voltage applied to the high voltage dome and the extraction voltage applied to the source. For quantitative comparison between the measurements and simulations, 


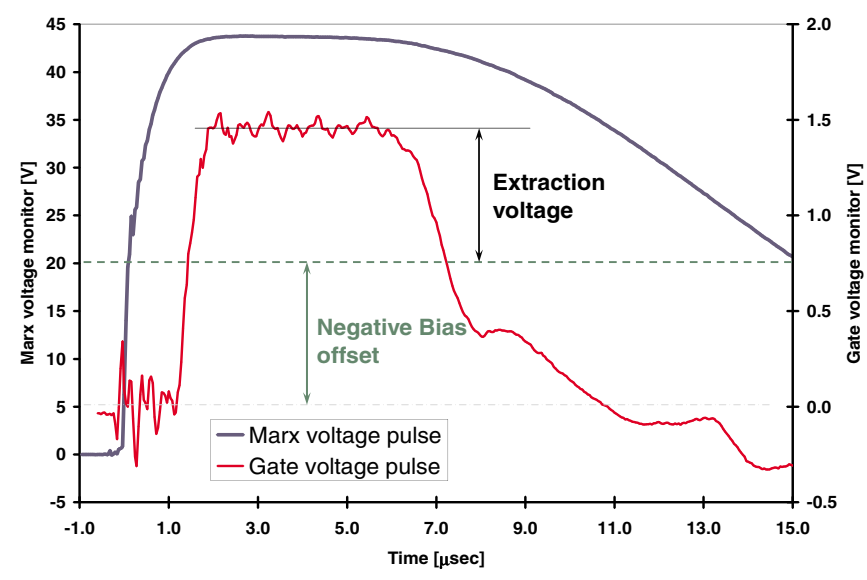

FIG. 3. (Color) Typical Marx voltage (blue) and gate voltage (red) pulses. The Marx voltage is viewed through a capacitive monitor, whose gain is $21.4 \mathrm{kV} / \mathrm{V}$. The gate voltage is viewed through a resistive divider and an optical link. The negative dc bias offset and net extraction voltage are indicated. Ripples in the extraction pulse waveform represent electrical noise in the signal.

accurate calibration of the dome and extraction voltage monitors is essential.

A capacitive dome voltage monitor is flush mounted on the wall of the pressure vessel. The dome voltage monitor was calibrated by applying a known pulsed high voltage to the dome. The calibration was refined by beam energy measurements in HCX using time of flight and an electrostatic energy analyzer. These measurements indicate a beam energy about $2 \%$ lower than expected from the capacitive voltage monitor, with absolute accuracy $\pm 2 \%$.

The extraction voltage pulse is monitored by a voltage divider, and the signal is transmitted through a fiber-optic link. This arrangement has proven difficult to calibrate because of uncertainties and drifting in the optic link, high-speed fiber-optic transceivers, and associated electronics. A calibration pulse train (1 pulse per second of known amplitude) is applied to the circuit at the dome to reduce uncertainty due to drifting. However, to minimize uncertainties, a new extraction voltage pulse calibration procedure was developed, utilizing the beam current and the dc bias and extraction pulser power supply voltages.

The beam current is measured by the downstream current transformer. The reproducibility of the current measurement is $1 \%$. The stability of the power supply voltage monitor and low-frequency fiber-optic circuit is also about $1 \%$.

In an ion diode, if source emission is sufficient, beam current is limited by the Child-Langmuir current $I$ :

$$
I=K_{\mathrm{gun}} V^{3 / 2},
$$

where $K_{\text {gun }}$ is the perveance of the ion gun and $V$ is the accelerating voltage. The perveance is a constant dependent only on the geometry of the diode and the mass and charge state of the ion. We have utilized this fact to produce an improved calibration procedure.

In this calibration procedure, we measure the beam current for various extraction pulser charging power supply voltages and bias power supply voltages. For those combinations of the measured voltages $V_{\text {gate }}$ and $V_{\text {bias }}$ which provide a given beam current $I_{b}$, we generate a series of linear least-squares regression fits to the data:

$$
V_{\text {gate }}=\alpha V_{\text {bias }}+\beta, \quad \text { for } I_{B}=\text { const. }
$$

The calibration constant is the slope of the linear regression at a fixed current $I_{B}$ :

$$
\alpha=\frac{\Delta V_{\text {gate }}}{\Delta V_{\text {bias }}} .
$$

The calibration constant $\alpha$ multiplied by the extraction pulser charging power supply voltage is the empirical value for the amplitude of the gate voltage pulse $V_{\text {gate }}$. This method greatly reduces drift in the measurement, and the gate voltage is determined to an accuracy of $\sim 2 \%$. Figure 4 illustrates an example of the calibration procedure. The predicted calibration factor is nearly independent of beam current when there is no scraping expected in the ESQ section.

Since the extraction voltage is the difference of two large voltages, it remains less well determined than the dome voltage. Measurements (Figs. 7-13) taken before the development of the improved calibration technique are based on the conventional calibration procedure through the high-speed fiber-optic chain and have a higher degree of uncertainty in the extraction voltage.

\section{Ion source}

The ion source consists of a porous tungsten hot plate substrate in either of two configurations. Some measurements were made using the substrate as a contact ionizer [12]. A potassium carbonate solution applied to the surface provided the reservoir of $\mathrm{K}^{+}$ions, which was depleted on a relatively short time scale of $\sim 20 \mathrm{~h}$. An aluminosilicate ion source with a considerably longer operational lifetime was also used. In an aluminosilicate ion source the substrate is coated with a potassium aluminosilicate material [13] which acts as the source of $\mathrm{K}^{+}$ions. There has been some concern about the uniformity of emission from this type of source. However, both types of sources yielded comparable beam characteristics during their operational lifetime, based on measurements downstream of the injector. An aluminosilicate source provided an operational lifetime of about $1 \mathrm{yr}$. The end of useful operational lifetime occurs when emission at the source is sufficiently nonuniform to perturb the beam current and envelope parameters downstream of the injector.

The source is concave to provide an initially convergent beam. It is heated by a filament to $T \approx 1100{ }^{\circ} \mathrm{C}$ and the extracted $\mathrm{K}^{+}$beam current density is space-charge limited 
to $j \leq 10 \mathrm{~mA} / \mathrm{cm}^{2}$. The intrinsic beam emittance at the source of radius $r=5 \mathrm{~cm}$ for $\mathrm{K}^{+}$ions of mass $M$ is $\varepsilon_{n}=$ $2 r \sqrt{k T / M}=0.18 \pi \mathrm{mm}$ mrad, where the normalized edge emittance is defined as 4 times the rms emittance, $\varepsilon_{n}=$ $4 \beta \gamma \varepsilon_{\mathrm{rms}}$, or

$$
\varepsilon_{n}=4 \beta \gamma \sqrt{\left\langle(x-\langle x\rangle)^{2}\right\rangle \cdot\left\langle\left(x^{\prime}-\left\langle x^{\prime}\right\rangle\right)^{2}\right\rangle-\left\langle(x-\langle x\rangle) \cdot\left(x^{\prime}-\left\langle x^{\prime}\right\rangle\right)\right\rangle^{2}}
$$

in the transverse dimension $x$ and similarly in $y, \beta$ and $\gamma$ are the usual relativistic factors, and the bracket notation indicates an average over the particle distribution.

Although the injector is capable of delivering beams of energy up to $2 \mathrm{MeV}$, the maximum beam energy for HCX is $1.8 \mathrm{MeV}$. The data presented here were for the most part

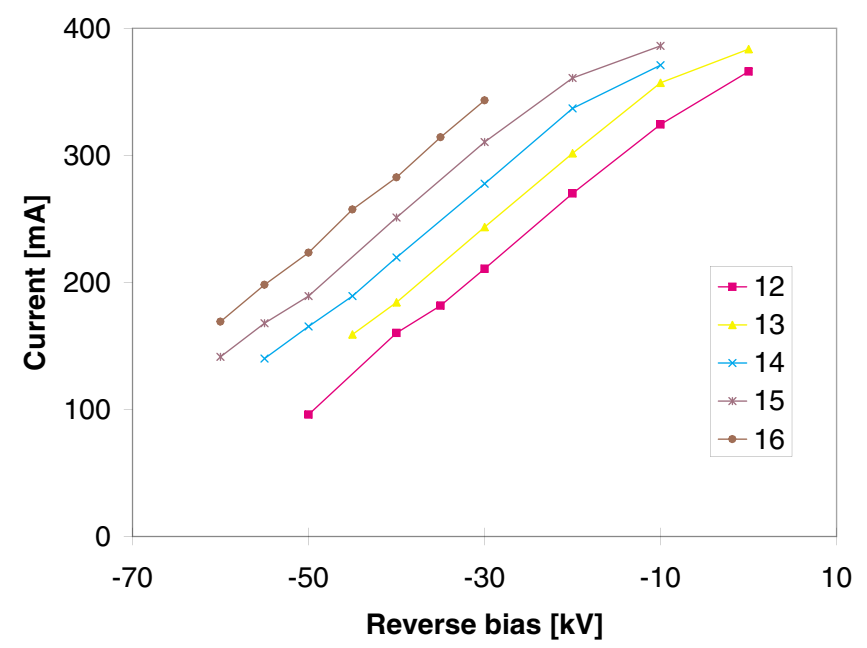

(a)

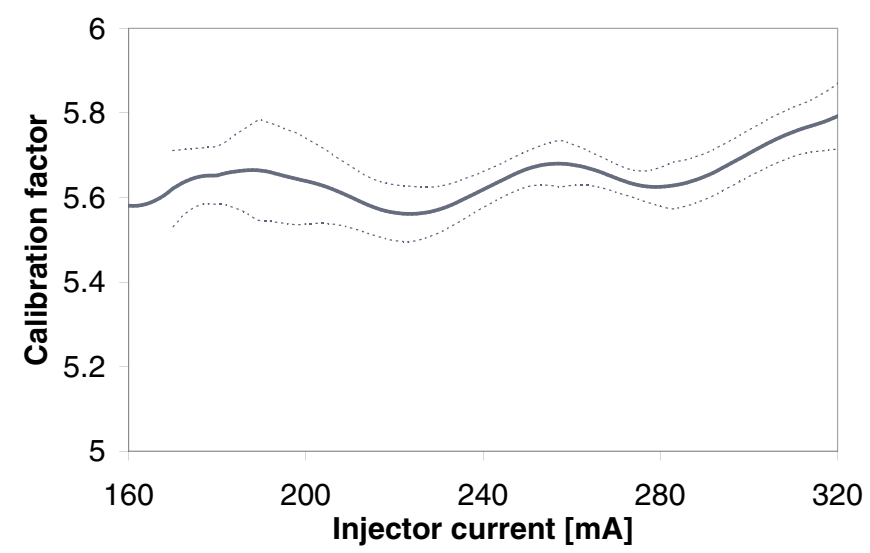

(b)

FIG. 4. (Color) (a) Measured beam current as a function of dc reverse bias voltage for extraction power supply voltages of 12 $16 \mathrm{kV}\left(V_{\mathrm{Marx}}=940 \mathrm{kV}\right)$. The slope of a least-squares linear regression through the points of (a) as a function of beam current provides the predicted calibration factor shown in (b). The calibration factor is the ratio of the amplitude of the gate voltage pulse to the extraction power supply voltage. The dashed lines indicate the standard error of the linear regression. At $180 \mathrm{~mA}$ the predicted calibration factor is 5.65 and the standard error is 0.07 or $1.2 \%$. taken in the range $0.97-1.5 \mathrm{MeV}$, with limited operation up to $1.8 \mathrm{MeV}$. The $\mathrm{K}^{+}$beam current at the most recent operating point is $183 \mathrm{~mA}$ at $0.97 \mathrm{MeV}$ and $462 \mathrm{~mA}$ at $1.8 \mathrm{MeV}$.

\section{E. Diagnostics and modeling}

Most of the beam diagnostics [14] used for commissioning the modified injector were located in a diagnostic end station at the exit of the injector. They include a current transformer (Rogowski coil) and a Faraday cup to measure the total current transported downstream, and a double-slit scanner to measure two-dimensional transverse beam distributions in $x, x^{\prime}$ and $y, y^{\prime}$ space. A 32-channel Faraday cup array (FCA) was used to characterize the transverse beam distribution exiting the diode. This diagnostic was inserted into the bore of the ESQ accelerator section from the downstream end. The 32 collectors were oriented in an L-shaped array; the assembly was rotated to map out the beam distribution and moved along the beam axis. Kapton films for imaging the beam were inserted at various locations in the injector. After HCX experiments began, the downstream diagnostic station was replaced by the HCX matching section, and the only injector beam diagnostic that remained available was the downstream current transformer. Assuming adequate suppression of electron currents, the absolute accuracy of beam current measurements is about $2 \%$.

First and second moments were extracted from the phase-space data from the double-slit scanner [8]. These moments determine the beam envelope sizes $a, b$ and divergence angles $a^{\prime}$ and $b^{\prime}$, defined as twice the rms beam size and divergence angle (corresponding to the beam size and divergence at the edge of a uniform distribution) and the edge emittance. An amplitude cut of about $4 \%$ of the maximum signal was applied to remove electrical noise from the calculation. Statistical uncertainty in the emittance measurement was about $10 \%$, but systematic uncertainty due to instrumental effects can be larger.

The limiting resolution of a double-slit emittance scanner is determined by the geometry of the slits and the drift distance. The scanner consists of a pair of slits of width $w=0.005 \mathrm{~cm}$ separated by a drift distance $d=15 \mathrm{~cm}$. In the absence of misalignments, angular resolution of the slit pair is limited by the ratio of the slit width to the drift distance. Instrumental broadening due to the finite width of the slit for a beam of radius $r=60 \mathrm{~mm}$ may be estimated by $\varepsilon_{n}=\frac{1}{2} \beta \frac{w r}{d}=0.14 \pi \mathrm{mm}$ mrad at $1 \mathrm{MeV}$. Instrumental broadening is increased by rotational misalignment be- 
tween the planes of the two slits and between the plane of the slits and the orientation of the beam ellipse, both of which in effect broaden the width of the slits. These effects were modeled by a Monte Carlo calculation. Since the horizontal and vertical scanners use different pairs of slits, instrumental broadening can be different, depending on the degree of misalignment in the respective planes. Instrumental broadening is particularly important at the exit of the injector because the beam is cold at this location, and in some cases the measured emittance may be dominated by instrumental broadening due to slit misalignment. The beam is matched into HCX by compressing transversely; therefore it develops a broader velocity distribution downstream of the injector and instrumental broadening is much less significant.

The injector has been designed and modeled by numerical simulation codes. The ion diode optics and beam parameters in the diode region were calculated by the particle trajectory code EGUN [15]. A PIC code, WARP [16], which is a fully three-dimensional time-dependent beam simulation code that includes the full physical geometry and any time dependencies in the fields, was used to simulate the beam in both the diode and ESQ sections of the injector. Recent improvements such as adaptive mesh refinement [17] have improved the reliability of WARP in handling aberrations especially near the source and the edge of the beam.

\section{BEAM CHARACTERIZATION}

Previous characterizations of the beam produced up to $0.8 \mathrm{~A}$ of $\mathrm{K}^{+}$ion beam at $2 \mathrm{MeV}$ using a $17-\mathrm{cm}$ diameter source. Measurements were made of the beam radius, beam centroid, and emittance of the exiting beam, and compared with WARP simulations [5]. The beam current density distribution was hollow, introducing significant space-charge nonuniformities and nonlinear self-fields. When injected into a linear transport channel, such distributions can lead to wave propagation and emittance growth.

In the present investigation, we have reduced the source diameter from 17 to $10 \mathrm{~cm}$. The smaller source has reduced intrinsic emittance, is easier to fabricate, and requires less heating power because of its smaller surface area. This modification also reduced the beam current from 0.8 to $0.6 \mathrm{~A}$.

Data from two configurations of the injector with a 10$\mathrm{cm}$-diameter source are presented below. In the initial configuration the beam underwent large transverse envelope oscillations leading to emittance growth through the ESQ section. In the second configuration the injector was optimized by modifying the diode section and retuning the ESQ section to reduce the beam oscillations, resulting in significant emittance reduction.

The first configuration used the smaller source and Pierce structure but maintained the previously established diode and ESQ conditions. The nominal voltage drops across the resistor tap points were (measured across the diode, Q1, Q2, Q3, and Q4, respectively) 670, 250, 350, 300 , and $350 \mathrm{kV}$. The beam was characterized at both the diode exit and at the exit of the injector. The beam envelope passes relatively close to the ESQ electrodes, as shown in a WARP simulation in Fig. 5 for the nominal $80 \mathrm{kV}$ equivalent extraction voltage (scaled to $V_{\text {beam }}=2.0 \mathrm{MeV}$ ). Normalized emittance increases to $0.3 \pi \mathrm{mm} \mathrm{mrad}$ as the beam leaves the source, due to aberrations in the diode. The normalized edge emittance also exhibits jumps in emittance due to transverse distortions as the beam approaches the ESQ electrodes.

In the second configuration, we modified both the diode geometry and the ESQ voltage distribution. To improve the diode optics, the gate electrode aperture diameter was decreased from 179.5 to $110 \mathrm{~mm}$, as in Fig. 1. The graphite Pierce structure was replaced with a water-cooled copper structure. To improve the ESQ section optics, the voltage drops in the resistive divider were modified to 595, 249, 368,276 , and $240 \mathrm{kV}$. These changes, based on a WARP model, increased the clearance between the edge of the beam and the ESQ electrodes in the accelerating column. The beam envelope and edge emittance are shown in Fig. 6 for the design extraction voltage. Emittance growth through the ESQ section is greatly reduced from the first configuration.

\section{A. Diode}

In order to characterize the beam exiting the diode, we isolated and energized the diode section only. The FCA diagnostic was inserted in the bore of the ESQ channel with the detector array at a location $23 \mathrm{~cm}$ downstream of

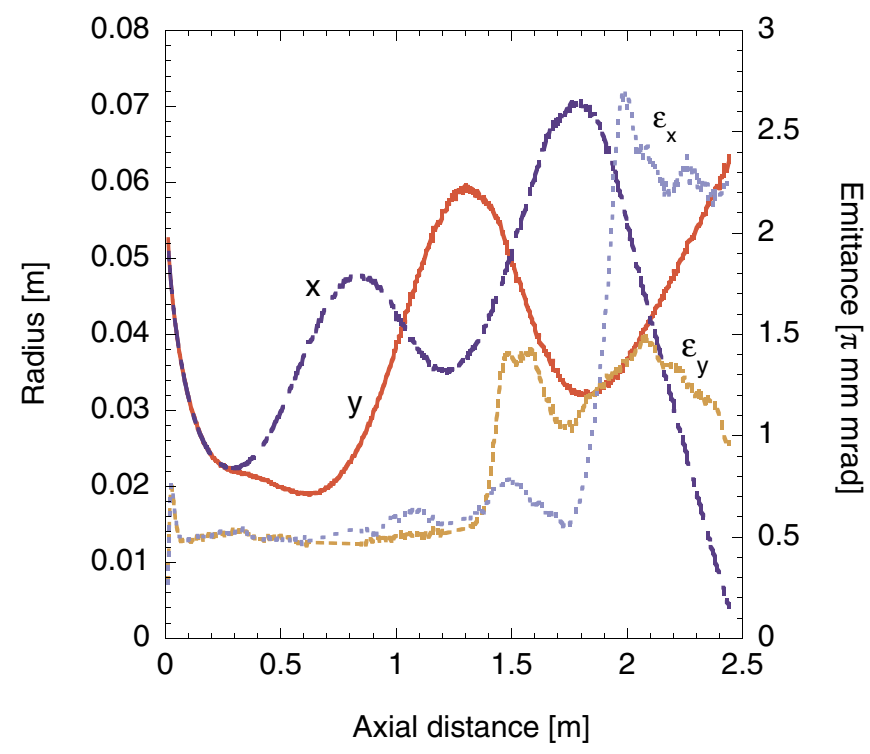

FIG. 5. (Color) Beam envelope (2 rms) and normalized edge emittance evolution in ESQ section for the first configuration. 


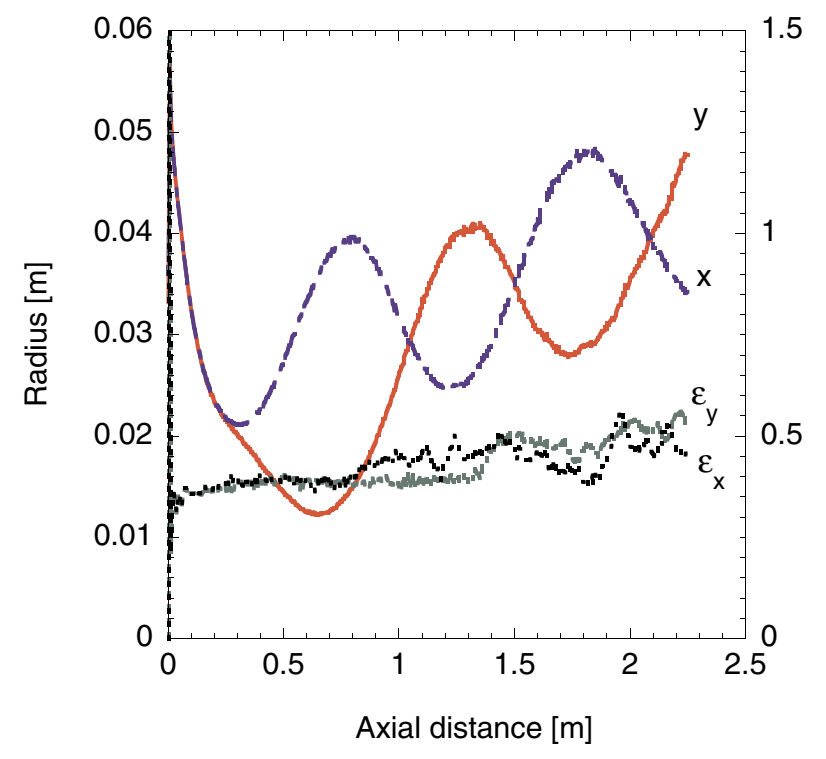

FIG. 6. (Color) Beam envelope and normalized edge emittance evolution in ESQ accelerator at the design point after modification for the second configuration.

the diode exit. An example of the current density map at the diode exit as measured by the FCA is shown in Fig. 7. These maps were generated for a number of cases. In Fig. 8 radial slices of the measured beam profiles are shown together with WARP results for three extraction voltages.

Two features to be noted in the current density map are the central peak and the rim near the edge. The central peak represents $<1 \%$ of the total beam current in this data. The central peak, which is not in general seen in the simulations, may reflect a perturbed space-charge distribution when the ion current is limited by source emission below

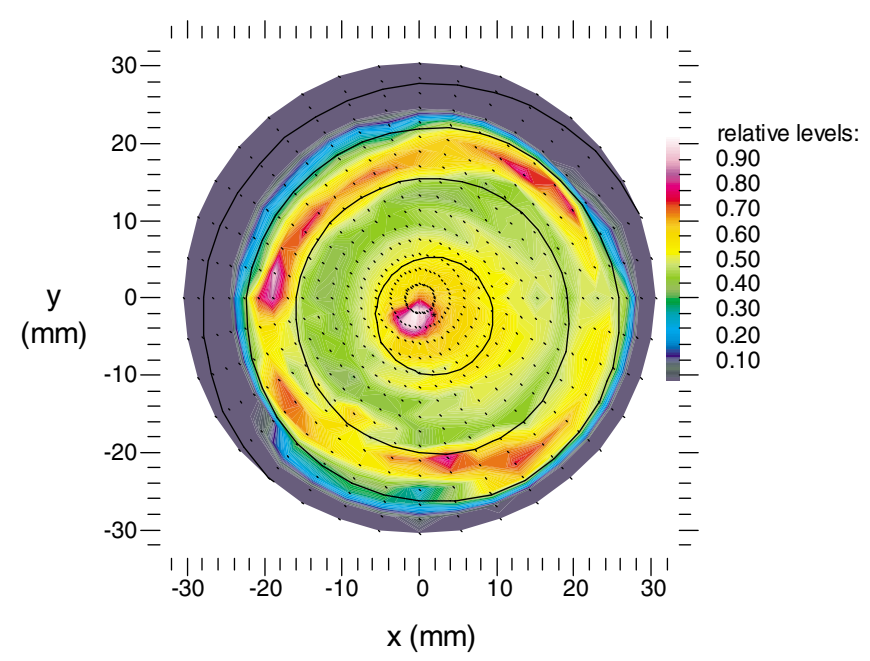

FIG. 7. (Color) Contour map of the beam current density profile $2.5 \mathrm{~cm}$ downstream of the extraction electrode for the $10-\mathrm{cm}$ diameter contact-ionization source. Intensities are the mean of a $1.2 \mu \mathrm{s}$ window taken in the middle of the pulse. The data points are on a polar grid with a radial spacing of $1.9 \mathrm{~mm}$.

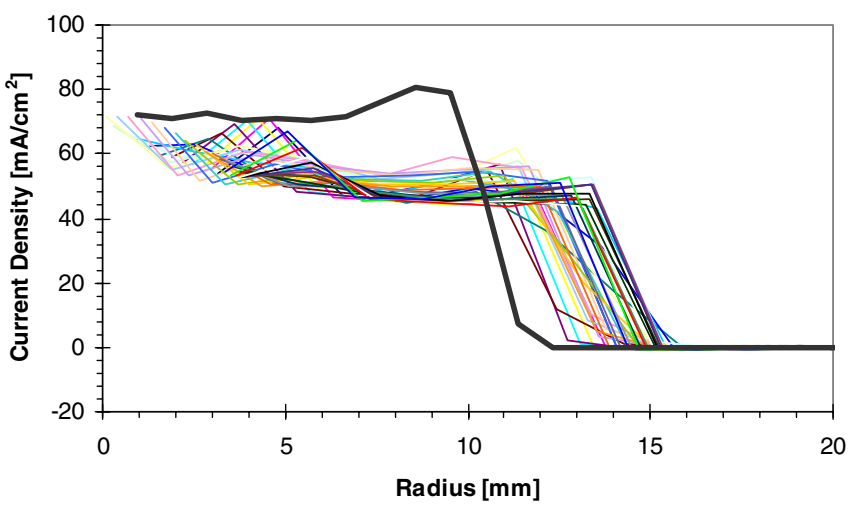

(a)

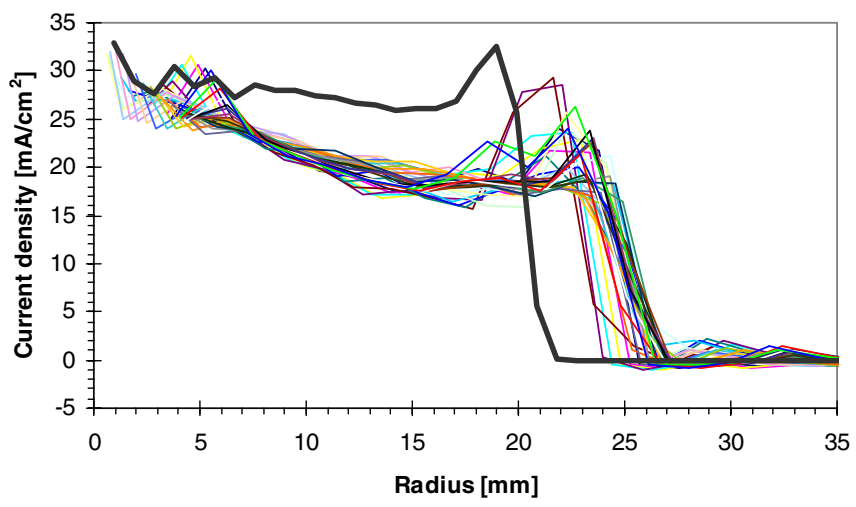

(b)

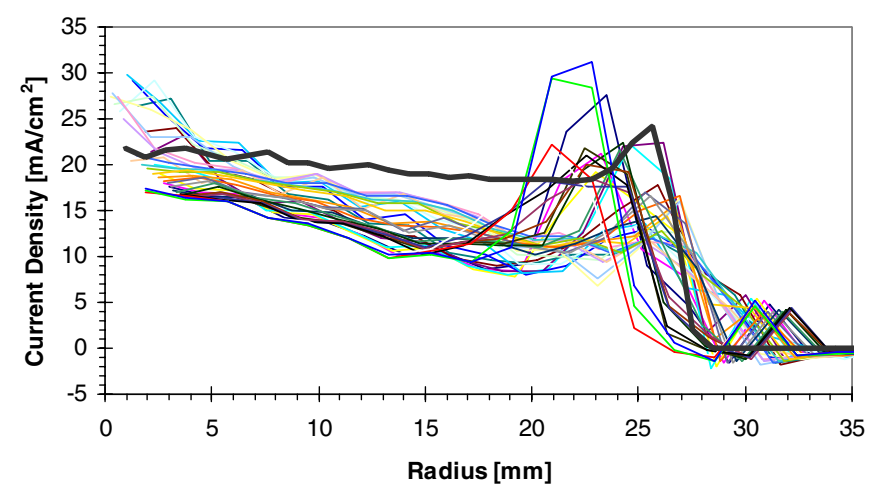

(c)

FIG. 8. (Color) Comparison of the measured and predicted radial beam profiles at $V_{\text {gate }}=0,31$, and $53.5 \mathrm{kV}$, respectively, for a fixed Marx voltage of $509 \mathrm{kV}$. The thick black curve is the prediction from the WARP PIC code.

the space-charge limit, or when electrons from the surface of the FCA may be entering the diode region where they can provide partial space-charge neutralization. The central peak under these conditions increases in amplitude during the pulse and is affected by the suppressor voltage and the ambient pressure in the diode, consistent with the presence or formation of a gas cloud on the surface of the collector array that acts as a source of electrons [18]. The rim near the edge of the beam in the simulations is due to aberrations originating near the outer edge of the source. 
The height of the rim increases as extraction voltage increases (Fig. 8). The general features of the measured beam profiles, in particular, the gentle positive slope in the core of the beam, the relatively abrupt edge, and the presence of the rim, are reproduced in the simulations. The measured vertical and horizontal 2 -rms beam sizes agree with each other to within $<1 \mathrm{~mm}$ and are shown in Fig. 9 together with simulation results for the conditions measured. The disagreements in beam size between the model and the data range up to $5 \mathrm{~mm}$.

The abrupt edge in the simulations was confirmed by time-integrated beam images on kapton film (Fig. 10). The kapton film was exposed to the beam at the bottom of a Faraday cup, $23 \mathrm{~cm}$ downstream of the diode exit, and shielded by honeycomb (expanded metal) material. Kapton has excellent spatial resolution and indicated a welldefined edge in the beam current density profile, which falls off over a radial distance of 3-5 mm. The kapton images, in contrast to the WARP model and the FCA data, showed little or no rim on the beam at this location.

Figure 11 shows a comparison between beam currents measured by a Faraday cup at the diode exit and predicted beam currents. The data points are normalized in terms of perveance because both the Marx and extraction voltages were varied over a wide range; the Marx voltage (which energizes the diode only) was varied from 390 to $679 \mathrm{kV}$ and measured beam currents ranged from 24 to $764 \mathrm{~mA}$. The relative scaling of the data as a function of extraction voltage is consistent with the expectations based on the simulations and with space-charge limited flow. Emission

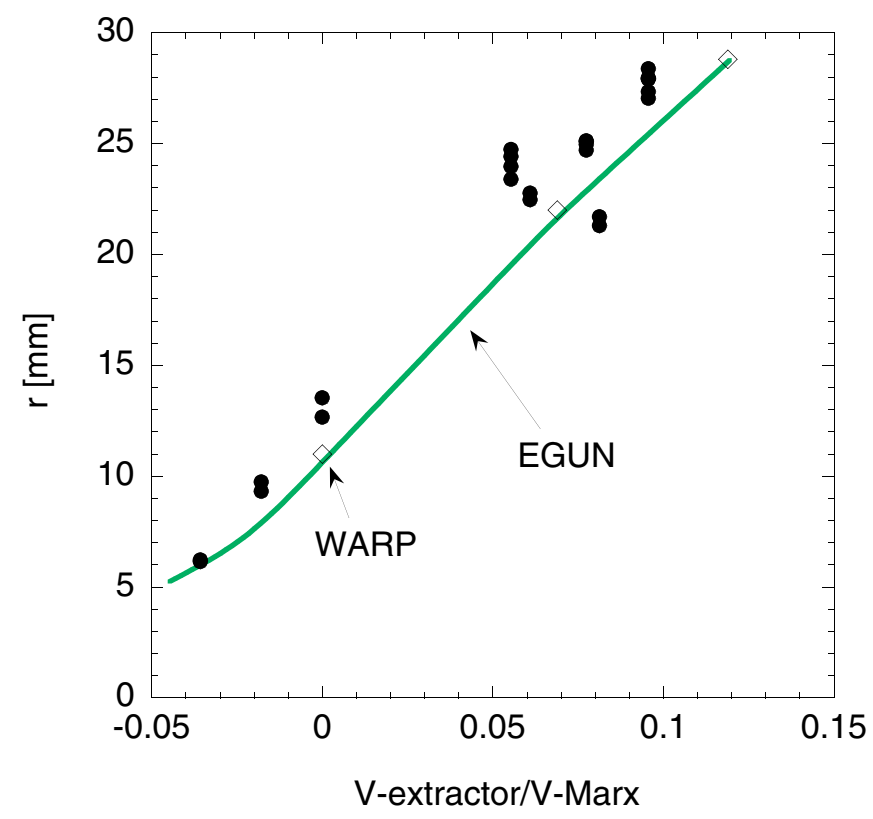

FIG. 9. (Color) Comparison of the 2-rms beam size (solid circles) measured at $2.5 \mathrm{~cm}$ downstream of the extraction electrode with EGUN (curve) and WARP (open diamonds) results as a function of the ratio of extraction voltage to Marx voltage.

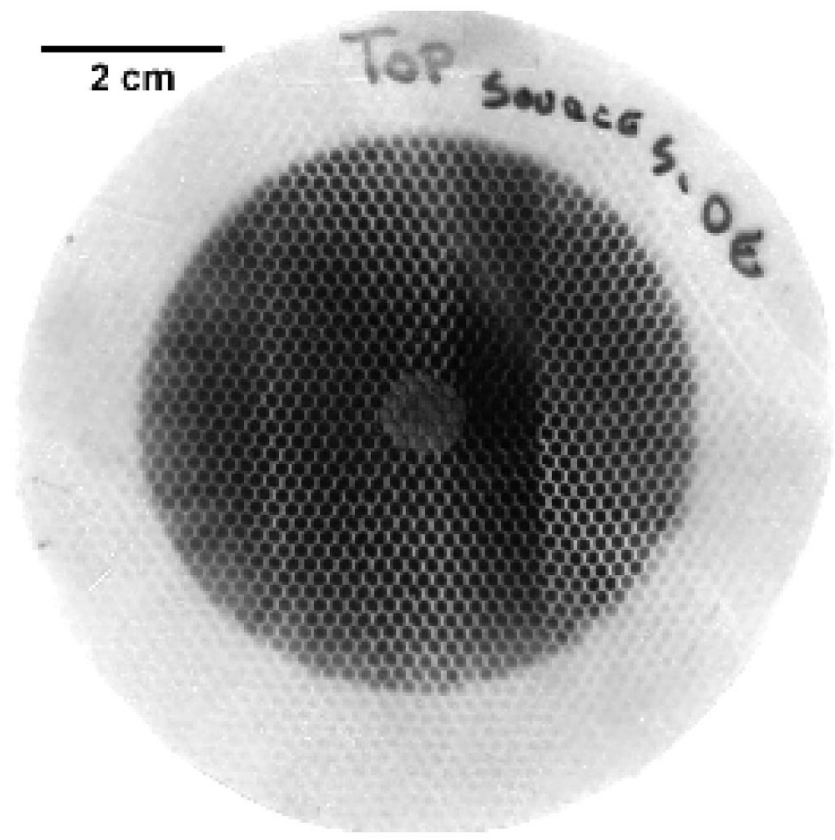

FIG. 10. Kapton image of beam exiting the diode. The regular patterns superimposed on the image are due to the honeycomb shielding structure.

limited current at the source would limit the beam below the predicted scaling with voltage, and would thus appear on this plot as a spreading of the data points. The discrepancy with respect to the predicted curve is $5 \%$ for one data set and 20\% for the other data set. A 20\% discrepancy is significant and may be due to miscalibration or incomplete suppression of electron currents in this case.

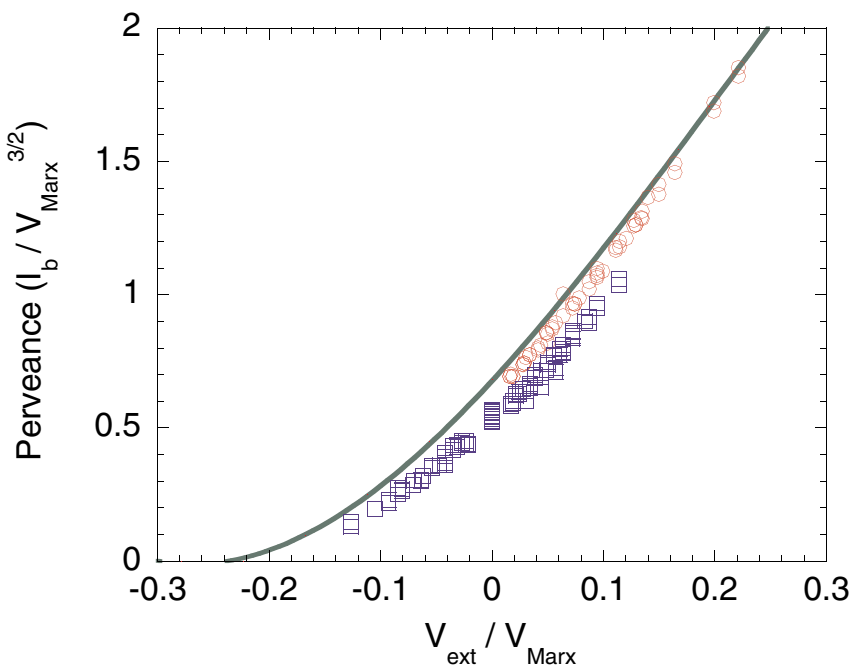

FIG. 11. (Color) Comparison with EGUN model of perveance (in units of $10^{-9} \mathrm{~A} / \mathrm{V}^{3 / 2}$ ) representing current exiting the diode as measured by a Faraday cup. The circles and squares represent data taken on two separate days, showing the level of day-to-day reproducibility in the measurement. 


\section{B. ESQ}

Kapton images of the beam exiting the injector in the first configuration [Figs. 12(a)-12(c)] show a timeintegrated beam current density profile which is rectangular in shape with a raised outer rim. As the extraction voltage is increased from $40 \mathrm{kV}$ (equivalent at $2 \mathrm{MeV}$ ) to the nominal $80 \mathrm{kV}$, the size and aspect ratio of the beam profile remain roughly constant, but the beam image evolves from elliptical to become increasingly rectangular in shape. A comparison with a WARP simulation [Fig. 12(d)] for the 80-kV configuration [12(a)] shows qualitative agreement with respect to the distortion of the beam ellipse, and the presence of a raised outer rim. The axial location of the WARP beam profile was chosen to match the measured aspect ratio of the beam. However, the observed size of the beam is significantly larger than in the simulation. The rim was not observed on kapton images taken at the midpoint of the ESQ section $(\mathrm{Q} 2$, at the position labeled "electrode plate" in Fig. 1). The internal fine structure in the images of Fig. 12 is related to transients at the head and tail of the beam pulse. The deviation from an elliptical shape is an indication of the degree of distortion due to aberrations in the ESQ section. The measured exit beam envelope parameters and normalized emittance (in units of $\mathrm{mm} \mathrm{mrad}$ ) at the location of the double-slit diagnostic $(z=2.42 \mathrm{~m}$ for horizontal measure- ments and $z=2.44 \mathrm{~m}$ for vertical measurements) are compared with the WARP results in Table I for 40 and $80 \mathrm{kV}$, where $80 \mathrm{kV}$ is the nominal extraction voltage.

The beam is near a horizontal waist at the measurement location, and the WARP model tends to underestimate the horizontal beam envelope size at this location. The vertical envelope size and divergence are well predicted by the model. However, there is a large discrepancy between the measured and predicted vertical emittance. The discrepancy is consistent with misalignment of the vertical slit scanner. When the extraction voltage was reduced further away from the operating point (to $0 \mathrm{kV}$ ), the disagreement between data and simulation increased.

Figure 13(a), the kapton image of the beam for the second configuration, shows a more uniform beam current distribution than those of Fig. 12. Additionally, the overall size of the beam is more suitable for further manipulation downstream in the HCX. Time-dependent PIC simulations were performed and comparisons with the data [Fig. 13(b)] again show some qualitative agreement in the degree of distortion and the presence of the raised outer rim. However, the predicted beam size disagrees with the model by up to $30 \%$ (horizontal).

The effect of the modified geometry on the phase-space distribution is seen in Figs. 14(c) and 14(d). Hooking is significantly reduced compared to Figs. 14(a) and 14(b). The measured normalized emittance decreased after modi-

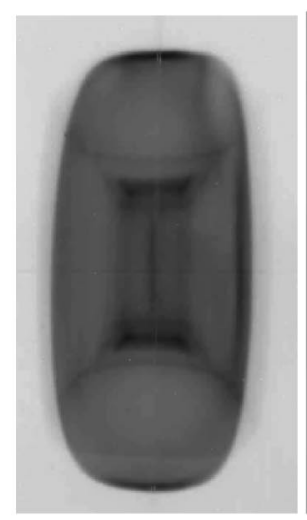

(a)

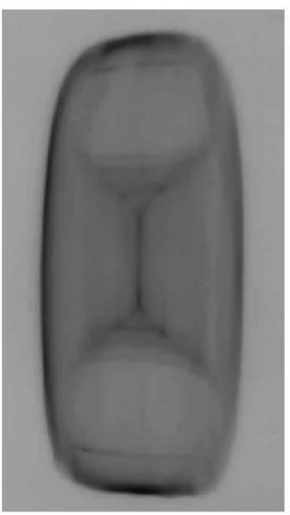

(b)

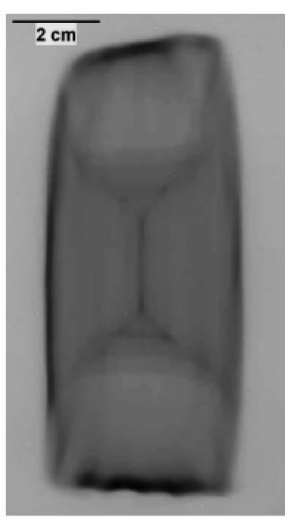

(c)

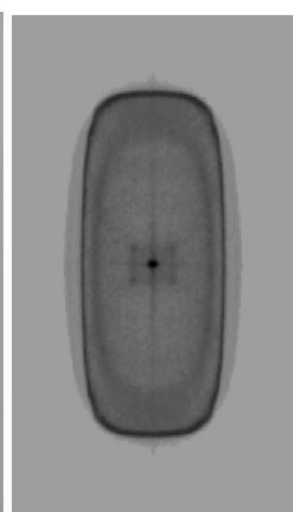

(d)

FIG. 12. Kapton film images of the beam taken at the exit of the injector (scale $=6.5 \times 11.5 \mathrm{~cm}$ ) at extraction voltages scaled to $2 \mathrm{MeV}$ (a) $40 \mathrm{kV}$, (b) $60 \mathrm{kV}$, and (c) $80 \mathrm{kV}$. (d) Corresponding time-integrated image obtained from wARP simulation (shown on the same scale). The fine structure seen in the center of the images is due to transients at the head and tail of the beam pulse.

TABLE I. Beam envelope parameters at exit.

\begin{tabular}{lcccccc}
\hline \hline Extraction voltage & $\varepsilon_{x, n}$ & $a$ & $a^{\prime}$ & $\varepsilon_{y, n}$ & $b$ & $b^{\prime}$ \\
\hline $80 \mathrm{kV}$ data & $2.2 \pi$ & $19.99 \mathrm{~mm}$ & $-88.4 \mathrm{mrad}$ & $1.9 \pi$ & $65.1 \mathrm{~mm}$ & $70.2 \mathrm{mrad}$ \\
$80 \mathrm{kV}$ WARP & 2.25 & 5.7 & -103 & 1.0 & 63.5 & 65.7 \\
$40 \mathrm{kV}$ data & 1.6 & 20.8 & -88.7 & $1.1 \pi$ & 60.5 & 71.3 \\
$40 \mathrm{kV}$ WARP & 2.1 & 6.6 & -103 & 0.7 & 61.6 & 69.1 \\
\hline \hline
\end{tabular}




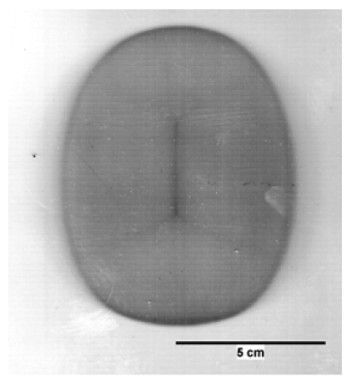

(a)

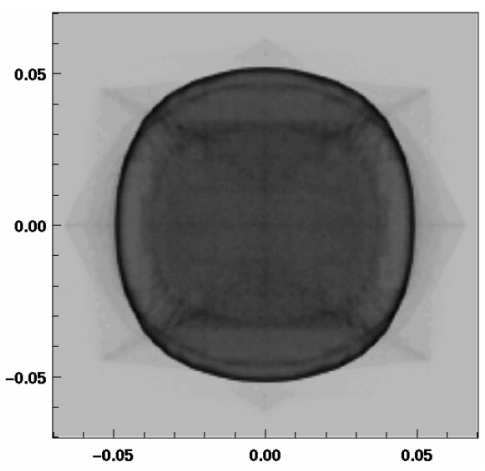

(b)
FIG. 13. Images of the beam at the exit of the injector. (a) Kapton film image taken after modifications were completed, (b) corresponding time-integrated image obtained from simulation (scales as shown).

fication, from $\varepsilon_{n}=1.9$ to $0.9 \pi \mathrm{mm} \mathrm{mrad}$ (vertical), reflecting the improved optics. The red ellipses show the ideal phase-space distribution limit for a uniform beam of equivalent rms size and emittance.

Unfortunately no data from the diode region is available for the second configuration. However, the transmitted current was measured by the downstream current transformer. Figure 15 shows measured and predicted beam currents over a range of extraction voltages. Figure 16 shows the ratio of the measured current to the predicted current. The predicted extraction voltage operating window for which the beam is expected to be transmitted without scraping the wall in the ESQ section is also shown. The observed region of good transmission corresponds well with the calculated region of good transmission. The average transmitted current is $95 \%$ of the predicted current, and all the data points fall within $92 \%-100 \%$ of the predicted value. The fraction of beam current transmitted falls off outside this window due to scraping, as expected. The transmission falls off gradually, especially on the high voltage side, before reaching the predicted edge of the window. The gradual falloff as the beam envelope approaches the wall may be due to the presence of a significant halo in the ESQ section.

The measured emittance at the exit $(0.5 \pi$ horizontal and $0.9 \pi$ vertical) is significantly larger than predicted $(0.5 \pi)$. The discrepancy is likely to be due at least in part to instrumental broadening, again in the vertical double-slit diagnostic. In fact, measurements downstream of the HCX matching section show that the emittance is $0.4 \pi$ horizontal and $0.5 \pi$ vertical [8]. Since there is little scraping observed and thus no reason to expect a reduction in emittance in the matching section, the actual emittance

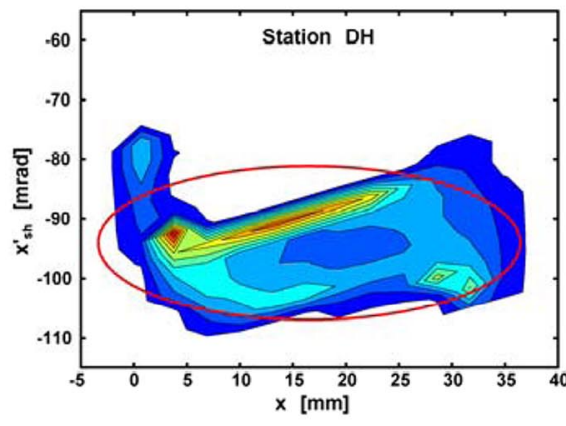

(a)

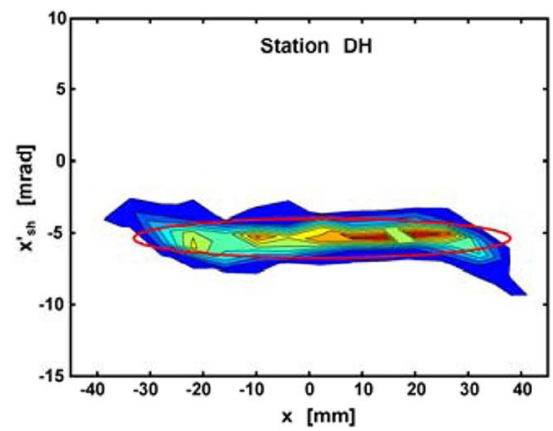

(c)

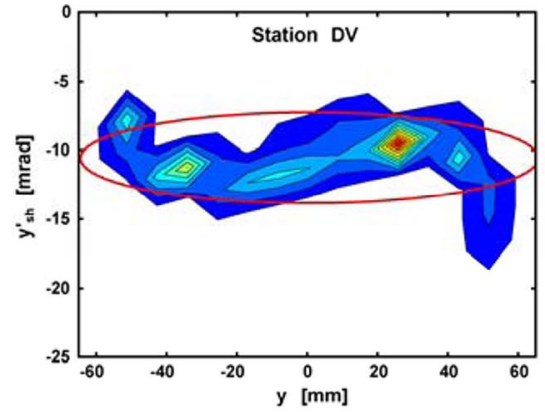

(b)

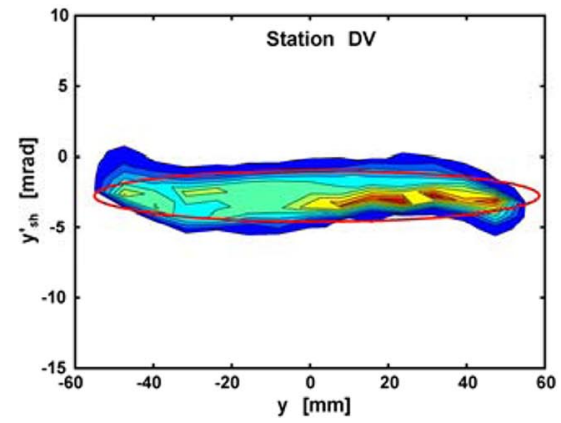

(d)

FIG. 14. (Color) Horizontal and vertical emittance diagrams measured at the exit of the injector. (a),(b) First configuration before modification of the beam optics, (c),(d) after modification of the beam optics. To emphasize distortions in these plots of "sheared" phase space, the phase space has been transformed to remove the envelope divergence $\left(a^{\prime}, b^{\prime}\right)$ of the beam. 


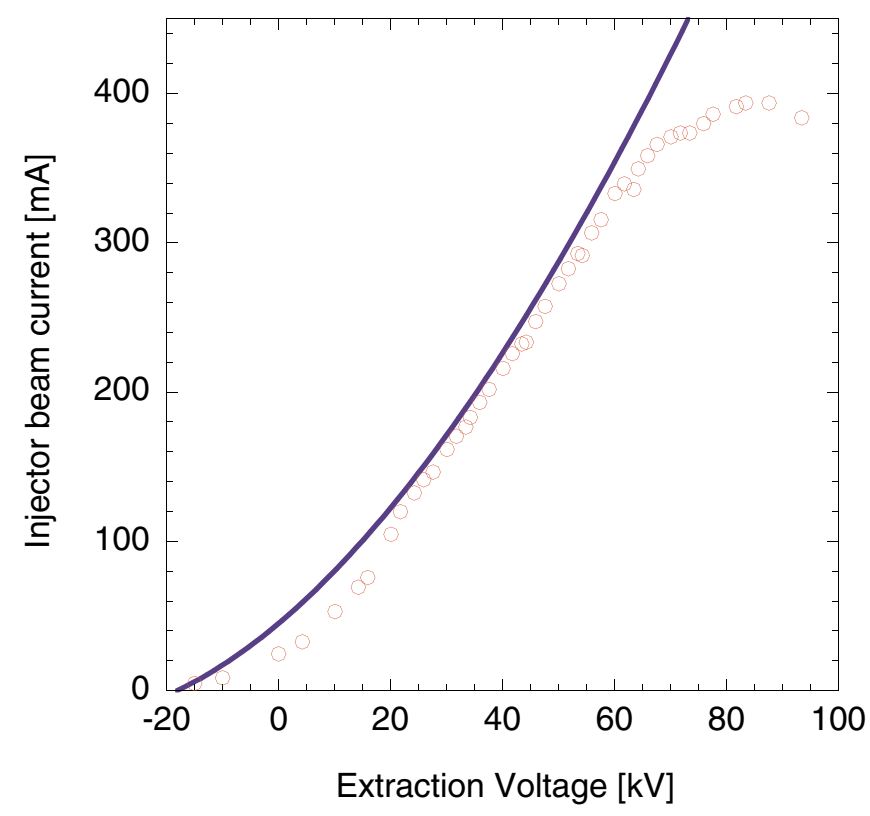

FIG. 15. (Color) Measured (circles) and predicted (curve) beam current downstream of injector as a function of extraction voltage for a fixed Marx voltage $\left(V_{\text {Marx }}=940 \mathrm{kV}\right)$ for final configuration.

exiting the injector must be less than or equal to the emittance exiting the matching section. The measured emittances exiting the matching section agree well with the WARP predictions.

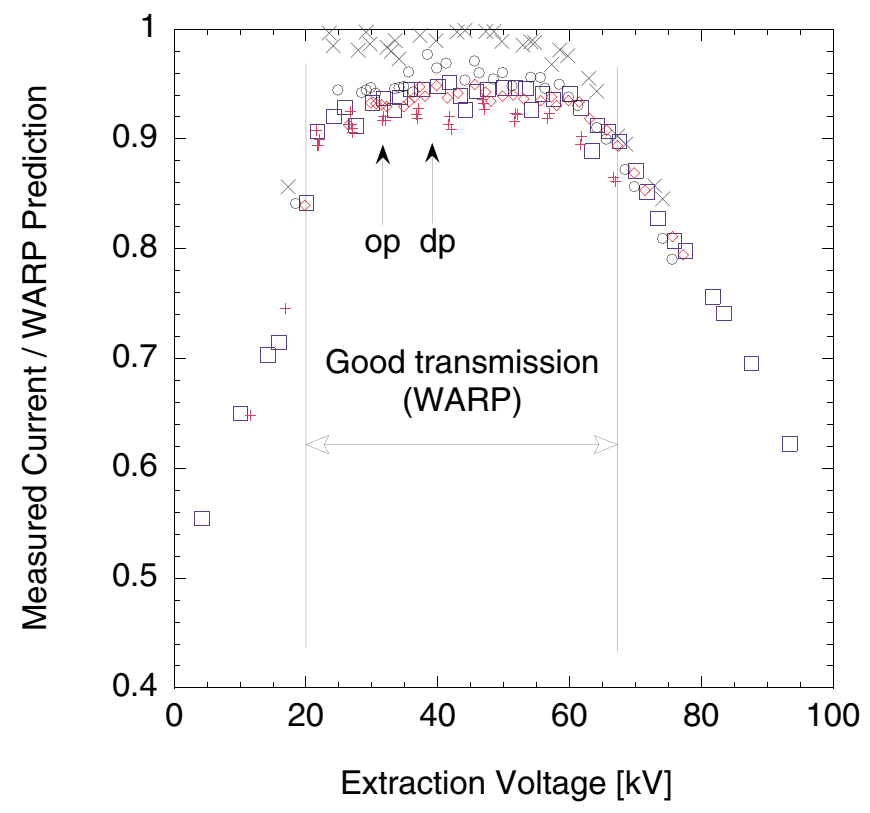

FIG. 16. (Color) Ratio of the beam current measured at the injector exit to the beam current calculated with WARP as a function of the extraction voltage. The predicted range of extraction voltages over which the beam should be transmitted without touching the wall is indicated. The operating point "op" and design point "dp" are indicated. Several series of measurements taken over a period of more than a year are shown.
The transient dynamics of the beam head has been simulated with a 3D time-dependent WARP model (Fig. 17). Note that with the existing rise time (a) the head of the beam shows a much more pronounced transient blowup (mushrooming) than with a reduced rise time (b) which is better matched to the beam transit time through the diode region. The scraping due to the blowup of the mismatched beam head generates secondary electrons and gas from the wall.

\section{DISCUSSION}

Requirements for the acceleration of beams with high charge density and low emittance have led to renewed interest in the ESQ accelerator [19]. We have performed detailed studies on the ESQ accelerator and demonstrated its utility as an injector with current and emittance adequate for HCX. The level of agreement between model and experiment reflects the level of our present understanding of beam dynamics in this configuration.

Allowing for experimental uncertainty the measurements are consistent with a beam current slightly smaller than (in nearly all cases 92\%-99\%) the current predicted by EGUN and WARP models. Qualitative features of the beam profiles exiting the diode are correctly modeled (not including the presence or absence of a raised rim), but the rms beam size, as well as details of the distribution, especially near the edge, are not accurately reproduced. The envelope parameters at the exit differ by as much as $30 \%$ from expectations. The simulations predict a larger beam radius than the measured radius. This discrepancy cannot be explained by beam current lower than predicted or by any partial space-charge neutralization in the ESQ channel. Both of these would tend to reduce the experimental beam size and increase the discrepancy between experimental and predicted beam sizes. There are several possible explanations for the discrepancy. Although the geometry of the solid-state source is simple, it cannot be reproduced perfectly due to limitations in fabrication techniques and thermal expansion. Beam profiles, including the presence or absence of a raised rim, are very sensitive to the detailed physical model of the diode region. Translational deformations of the surface of the source, extraction voltage errors, or imperfect emission can affect the beam profile, especially near the edge of the beam. Imperfections in the codes or in description of the beam conditions at the source surface are also suspect. These inconsistencies will be further explored with simulations and a dedicated source and injector test stand [20].

Beams in the initial configuration studied, which have an excursion near one or more of the ESQ electrodes, show distortions when exiting the injector, including emittance growth and a square-shaped distribution on exit. The large reduction in distortions and beam emittance in the second configuration when the envelope excursions are suppressed reflects the importance of careful design in the ESQ section 


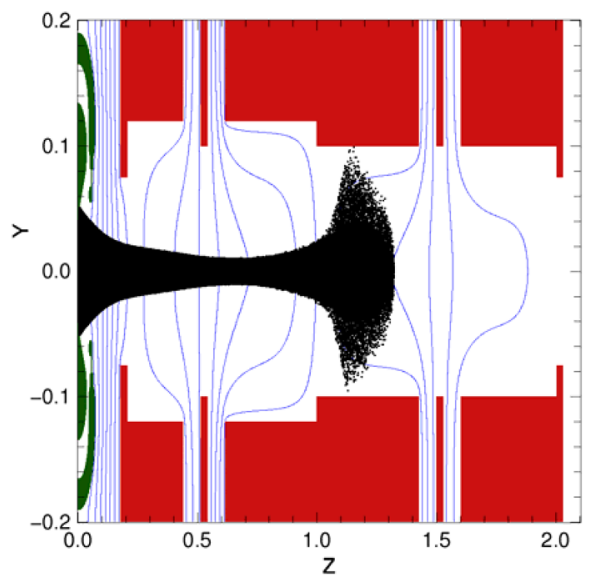

(a)

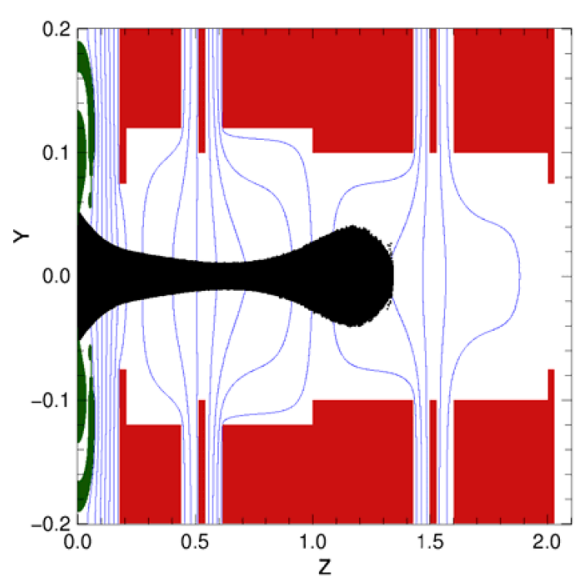

(b)

FIG. 17. (Color) Results from a time-dependent 3D PIC simulation showing the behavior of the head of the beam pulse according to the extraction voltage rise time. (a) Vertical shape of the beam head using the measured rise time of the voltage pulse, showing scraping of the beam on the ESQ electrodes. (b) Vertical shape of the beam head with rise time reduced by a factor of 2 , showing a more confined beam head.

of the accelerator. However, the beam emittance remains significantly larger than the intrinsic beam emittance due to thermal effects at the source.

A raised rim is clearly present at the exit of the ESQ section. An investigation of similar raised rims in an electron beam transport experiment shows transverse oscillations of the rim [21].

\section{ACKNOWLEDGMENTS}

The authors gratefully acknowledge the support of the scientific staff of the Heavy-Ion Fusion Virtual National Laboratory (HIF-VNL) and the outstanding assistance of R. Hipple and the LBNL technical staff. This work was performed under the auspices of the U.S. Department of Energy by University of California, Lawrence Livermore and Lawrence Berkeley National Laboratories under Contracts No. W-7405-Eng-48 and No. DE-AC0376SF00098 and the University of Maryland under Contracts No. DE-FG02-02ER54672 and No. DE-FG0294ER40855.

[1] C. M. Celata et al., Phys. Plasmas 10, 2064 (2003).

[2] E. A. Abramyan, M. M Brovin, V. V. Vecheslavov, V. A. Gorbunov, V.I. Kononov, and I. L. Chertok, Sov. At. Energy 29, 1089 (1970).

[3] J. W. Kwan, G. D. Ackerman, C. F. Chan, W. S. Cooper, G. J. de Vries, W. F. Steele, M. E. Stuart, M. C. Vella, R. P. Wells, T. Inoue, Y. Okumura, and M. Mizuno, Rev. Sci. Instrum. 66, 3864 (1995).

[4] J. W. Kwan, L. Ahle, D. N. Beck, F. M. Bieniosek, A. Faltens, D. P. Grote, E. Halaxa, E. Henestroza, W. B. Herrmannsfeldt, V. Karpenko, and T. C. Sangster,
Nucl. Instrum. Methods Phys. Res., Sect. A 464, 379 (2001).

[5] S. S. Yu, S. Eylon, E. Henestroza, C. Peters, L. Reginato, A. Tauschwitz, D. Grote, and F. Deadrick, Fusion Eng. Des. 32-33, 309 (1996); L. Reginato and C. Peters, Fusion Eng. Des. 32-33, 337 (1996).

[6] F. M. Bieniosek, E. Henestroza, and J. W. Kwan, Rev. Sci. Instrum. 73, 1042 (2002).

[7] J. W. Kwan, F. M. Bieniosek, E. Henestroza, L. Prost, and P. Seidl, Laser Part. Beams 20, 441 (2002).

[8] L. R. Prost, D. Baca, F. M. Bieniosek, C. M. Celata, A. Faltens, E. Henestroza, J. W. Kwan, M. Leitner, P. A. Seidl, W. L. Waldron, R. Cohen, A. Friedman, D. Grote, S. M. Lund, and A. W. Molvik, LBNL Report No. 53014, 2004.

[9] M. Lampel and M. Tiefenback, Appl. Phys. Lett. 43, 57 (1983).

[10] L. G. Christophorou, Nucl. Instrum. Methods Phys. Res., Sect. A 268, 424 (1988).

[11] A. W. Molvik, D. Baca, F. M. Bieniosek, R. H. Cohen, A. Friedman, M. Kireef-Covo, L. Prost, and P. A. Seidl, in Proceedings of the 2004 International Symposium on Heavy Ion Inertial Fusion, Princeton, NJ [Nucl. Instrum. Methods Phys. Res., Sect. A (to be published)].

[12] J. W. Kwan, F. M. Bieniosek, E. Chacon-Golcher, and D. Baca, in Proceedings of the Particle Accelerator Conference, Chicago, IL, 2001 (IEEE, Piscataway, NJ, 2001).

[13] D. Baca, J. W. Kwan, and J. K. Wu, in Proceedings of the Particle Accelerator Conference, Portland, OR, 2003 (IEEE, Piscataway, NJ, 2003).

[14] F. M Bieniosek, S. Eylon, A. Faltens, A. Friedman, J. W. Kwan, M. A. Leitner, A. W. Molvik, L. Prost, P. K. Roy, P. A. Seidl, and G. Westenskow, in Proceedings of the 2004 International Symposium on Heavy Ion Inertial Fusion, Princeton, NJ (Ref [11]).

[15] W. B. Herrmannsfeldt, Stanford Linear Accelerator Center Report No. SLAC-PUB 66729, 1994. 
[16] D. P. Grote, A. Friedman, I. Haber, and S. Yu, Fusion Eng. Des. 32-33, 193 (1996).

[17] J. L. Vay, P. Colella, J.W. Kwan, P. McCorquodale, D. B. Serafini, A. Friedman, D. P. Grote, G. Westenskow, J.C. Adam, and A. Heron, Phys. Plasmas 11, 2928 (2004).

[18] F. M. Bieniosek, J. W. Kwan, E. Henestroza, and C. Kim, Nucl. Instrum. Methods Phys. Res., Sect. A 464, 592 (2001).
[19] J. W. Kwan, O. A. Anderson, L. L. Reginato, M. C. Vella, and S. S. Yu, Nucl. Instrum. Methods Phys. Res., Sect. B 99, 710 (1995).

[20] J. W. Kwan, F. M. Bieniosek, W. L. Waldron, J. L. Vay, G. A. Westenskow, E. Halaxa, and I. Haber, in Proceedings of the 2004 International Symposium on Heavy Ion Inertial Fusion, Princeton, NJ (Ref. [11]).

[21] S. Bernal, R. A. Kishek, M. Reiser, and I. Haber, Phys. Rev. Lett. 82, 4002 (1999). 